\title{
Actualité documentaire
}

\section{Bernadette Plumelle}

\section{CpenEdition}

\section{Journals}

Édition électronique

URL : http://journals.openedition.org/ries/3655

DOI : $10.4000 /$ ries.3655

ISSN : 2261-4265

\section{Éditeur}

Centre international d'études pédagogiques

\section{Édition imprimée}

Date de publication : 15 avril 2014

Pagination : 7-11

ISBN : 978-2-85420-603-6

ISSN : 1254-4590

\section{Référence électronique}

Bernadette Plumelle, «Actualité documentaire », Revue internationale d'éducation de Sèvres [En ligne], 65 | avril 2014, mis en ligne le 15 avril 2014, consulté le 06 janvier 2020. URL : http:// journals.openedition.org/ries/3655; DOI : 10.4000/ries.3655

Ce document a été généré automatiquement le 6 janvier 2020

(c) Tous droits réservés 


\section{Actualité documentaire}

\section{Bernadette Plumelle}

1 BODIN Romuald, ORANGE Sophie

L'université n'est pas en crise : les transformations de l'enseignement supérieur : enjeux et idées reçues

Bellecombe-en-Bauges : Éditions du croquant, 2013, 213 p.

L'objet de ce livre est d'aller à contre-courant de prises de position sur l'université française. Les auteurs, sociologues, font la critique des discours négatifs tenus en opposant une vision plus objective de la situation actuelle de l'université. Ils proposent un état des lieux fondé sur les résultats d'enquêtes et sur l'exploitation de données statistiques nationales. Le premier chapitre est consacré à la présentation duale de l'enseignement supérieur, avec des filières sélectives accessibles sur concours ou sur candidature et des filières non sélectives. Les trois chapitres suivants s'intéressent aux trajectoires des étudiants issus de baccalauréats technologiques et professionnels (débouchés, décrochages, situations d'abandon et d'échec). Les auteurs montrent le ressenti psychologique de ces étudiants face au décalage entre leur culture d'origine et les attentes qu'ils rencontrent en licence, ainsi que la très forte attractivité que peut représenter l'université pour eux.

BRUCY Guy, MAILLARD Fabienne, MOREAU Gilles

(sous la direction de)

Les « petits » diplômes professionnels en France et en Europe

Cahiers de la recherche sur l'éducation et les savoirs, 2013, hors-série $n^{\circ}$ 4, 201 p.

À l'heure où la généralisation de l'accès à l'enseignement supérieur est considérée par l'Union européenne comme l'un des grands enjeux des programmes éducatifs, les auteurs de ce dossier s'attachent à considérer l'autre versant de cette politique, celui des «petits" diplômes professionnels. La première partie offre deux panoramas d'ensemble de la situation européenne avec, pour l'un, un inventaire des divergences et convergences observées dans huit pays européens (Allemagne, Écosse, Espagne, France, Irlande, Pays-Bas, Pologne, Roumanie et Slovénie) et, pour l'autre, une étude de l'influence de la politique européenne, qui oscille entre coordination des systèmes nationaux de formation et promotion d'un modèle de formation européen. La seconde partie décrit quatre "petits» diplômes en France (CAP), en Suisse (CFC, AFP) et en 
Allemagne. Le dossier se clôt sur trois articles articulant politique des diplômes et marché du travail.

CRISTALDI Melita

Les dimensions culturelles de l'apprentissage: la contribution de la psychomotricité interculturelle

Cooperativa universitaria editrice catanese di magistero/Catania, 2013, $124 \mathrm{p}$.

L'auteure présente le rôle spécifique de la psychomotricité interculturelle dans la compréhension de l'apprentissage et s'intéresse plus particulièrement à la psychomotricité en tant que pratique thérapeutique et éducative, à la lumière de l'interculturalité.

DUPRIEZ Vincent, MALET Régis (sous la direction de)

L'évaluation dans les systèmes scolaires: accommodements du travail et reconfiguration des professionnalités

Bruxelles : De Boeck Université, 2013, 193 p.

Durant ces vingt dernières années, les systèmes éducatifs ont développé une diversité de politiques et d'outils d'évaluation visant à renouveler les formes traditionnelles de contrôle du travail des enseignants. L'ouvrage traite des politiques d'évaluation, de leur réception et de leur traduction dans les établissements scolaires et par les personnels d'enseignement. Après deux textes théoriques, les parties suivantes explorent les transformations du travail éducatif dans les établissements scolaires. Au-delà de la rhétorique commune sous-jacente à ces évolutions, les auteurs font apparaître dans les différents pays étudiés (France, Suisse, Angleterre, États-Unis) une grande diversité de politiques et dispositifs d'évaluation selon les principes qui les animent (rendre des comptes aux usagers ou aux autorités publiques), en fonction du niveau d'intervention pris en compte et des sanctions ou incitations données. Les analyses font apparaître une multiplication de normes et de prescriptions avec l'addition de nouveaux dispositifs d'évaluation aux anciens dispositifs et de nouvelles formes de contrôle de travail, qui pèsent sur l'identité des enseignants.

ENDRIZZI Laure

\section{Les lycées à la croisée de tous les parcours}

Dossier de veille de l'IFÉ, ENS de Lyon, décembre 2013, n 88, 20 p. [en ligne]

Dans un contexte d'allongement de la scolarité, ce dossier examine les offres de formation dans le deuxième cycle de l'enseignement secondaire, ainsi que les enjeux propres à ce niveau d'études. Il s'appuie sur les travaux comparatifs de la Commission européenne et de l'OCDE et sur un état des lieux des réformes, récentes ou en cours, dans quelques pays. Il met au jour les équilibres plus ou moins fragiles entre une formation générale humaniste et une formation utile préqualifiante ou qualifiante. L'auteure propose une synthèse des enjeux propres au secondaire supérieur et rend compte des mécanismes de différenciation et des voies diverses de formation empruntées par les élèves (14-19 ans). Des monographies (Allemagne, Belgique, Canada (Québec), Espagne, Suède et Suisse) sont téléchargeables séparément.

\section{FRELAT-KAHN Brigitte}

\section{Pragmatisme et éducation : James, Dewey, Rorty}

Paris : Vrin, 2013, 205 p. (Philosophie de l'éducation)

Depuis la fin du XXe siècle, la théorie de J. Dewey et les développements contemporains du pragmatisme suscitent un fort intérêt en France. Le pragmatisme y a longtemps rencontré des réticences à cause de son caractère « américain » et de sa réduction à la 
pédagogie mais il rencontre aujourd'hui un écho et une influence inédits. Dans la première partie de l'ouvrage, deux caractéristiques centrales du pragmatisme sont dégagées : la récusation de toute forme de dualisme et l'affirmation du pluralisme. La partie suivante est consacrée à la critique du dualisme tant métaphysique que cognitif et la troisième partie explore les conséquences sur la philosophie de l'éducation. L'auteure montre comment s'articulent conception du savoir, théorie de la vérité et définitions logiques d'une part; pensée du politique, du pouvoir et de la démocratie d'autre part.

\section{MEURET Denis}

Pour une école qui aime le monde : les leçons d'une comparaison France-Québec : 1960-2012

Presses universitaires de Rennes, 2013, $207 \mathrm{p}$.

$\mathrm{Au}$ cours des années soixante, la commission Parent a proposé au Québec un modèle politique d'éducation, ou « récit ", global et positif sur l'école et son rôle dans la société moderne. À la même époque, en France, le colloque d'Amiens a proposé un récit moins ample et beaucoup plus ambivalent, même si, dans les deux cas, on réclamait une école moins rigide et plus ouverte. Dans une première partie, l'auteur montre l'évolution de l'état de l'école en France et au Québec entre la période actuelle et les années soixante à différents niveaux (efficacité, équité, bien être des élèves). La deuxième partie analyse l'élaboration des deux récits proposés en France et au Québec dans les années soixante. L'auteur compare ensuite le gouvernement de l'école en France et au Québec (l'élaboration des politiques, leur contenu, leur mise en œuvre), reliant la qualité de l'école à la qualité de son gouvernement et celle-ci à la qualité du récit qui la fonde.

8 TARDIF Maurice

La condition enseignante au Québec du XIX ${ }^{\mathrm{e}}$ au XXI ${ }^{\mathrm{e}}$ siècle : une histoire cousue de fils rouges : précarité injustice et déclin de l'école publique

Québec : Presses de l'Université Laval, 2013, 334 p.

Depuis plusieurs années, les enseignants de l'école publique au Québec semblent vivre une période particulièrement difficile de leur profession. L'ouvrage analyse l'évolution de la condition enseignante au Québec depuis le XIX siècle, afin d'en distinguer les tendances à long terme. Il articule trois niveaux d'analyse: les enseignants et leur profession, l'institution scolaire dans laquelle ils travaillent et la société qui définit, pour une bonne part, la nature de l'éducation et l'exercice de l'enseignement. L'auteur s'appuie sur des travaux anciens et récents en sciences sociales, ainsi que sur la documentation produite par des organismes éducatifs et gouvernementaux pour mieux comprendre les enjeux sociaux, politiques et idéologiques de la condition enseignante. Il porte un regard très critique sur la dégradation de la condition des enseignants au Québec, liée au déclin de l'école publique et à la montée de l'injustice scolaire.

YATES Lyn, GRUMET Madeleine (ed.)

World year book of education 2011: curriculum in today's world: configuring knowledge, identities, work and politics

Londres : Routledge, 2014, $254 \mathrm{p}$.

L'ouvrage réunit des contributeurs de différents horizons géographiques pour réfléchir à la façon dont les événements de la dernière décennie ont influencé le curriculum dans leur pays. Comment, par exemple, est abordé le nationalisme dans le contexte de la mondialisation économique ou la crise financière internationale? Comment sont représentés les droits des femmes et des groupes ethniques? Les chapitres sont 
organisés en quatre parties: liens entre le curriculum et les identités nationale/ mondiale; relations entre le curriculum et l'économie internationale et nationale; rapport entre curriculum et connaissance; exemples de curriculum dans des pays présentant des situations politiques particulières. Les auteurs proposent ainsi une nouvelle approche des curricula actuels. Comment le monde y est-il représenté ? Comment les réponses aux événements mondiaux façonnent-elles les histoires racontées aux élèves sur ce qu'ils sont et peuvent être?

Publications d'organisations européennes et internationales

Eurydice : réseau d'information sur l'éducation en Europe

Education and training in Europe 2020. Responses from the EU member states

Bruxelles : Eurydice, décembre 2013, 105 p., bibliogr., [en ligne]

Le rapport rend compte des réponses apportées par les pays de l'Union européenne aux priorités définies dans la Stratégie européenne pour 2020 en matière d'éducation et de formation. Il se concentre sur les réformes récemment engagées dans les domaines suivants: le décrochage scolaire précoce, la diplomation et la qualité dans l'enseignement supérieur, les compétences et l'enseignement professionnel pour l'insertion des jeunes, et la participation des adultes à la formation tout au long de la vie.

11 Eurydice : réseau d'information sur l'éducation en Europe

Towards a mobility scoreboard: conditions for learning abroad in Europe

Luxembourg: Office des publications de l'Union européenne, janvier 2014, 62 p. [en ligne]

Cette étude propose un jeu d'indicateurs pour un "tableau de bord de la mobilité » destiné à mesurer les conditions favorisant la mobilité des étudiants dans l'enseignement supérieur. À la suite d'une recommandation du Conseil européen de 2011, dans laquelle les États membres se sont engagés à promouvoir la mobilité, l'étude vise à établir un cadre de suivi des progrès. Le rapport couvre des sujets comme l'information et les conseils pour la préparation linguistique, les aides financières (bourses, prêts, subventions...), la reconnaissance des acquis et le soutien aux apprenants défavorisés. Ce tableau de bord de la mobilité concerne principalement l'enseignement supérieur (année 2011-2012) pour 33 pays (les 28 États membres de l'Union européenne et l'Islande, le Liechtenstein, la Norvège, la Suisse et la Turquie).

12 OCDE

Résultats du PISA 2012 : savoirs et savoir-faire des élèves. Performance des élèves en mathématiques en compréhension de l'écrit et en sciences (volume 1). Version préliminaire

Paris : OCDE, décembre 2013, 274 p. [en ligne]

Plus de 510000 élèves dans 65 pays et économies ont participé à l'évaluation du PISA 2012. Les mathématiques sont le domaine principal d'évaluation et les sciences et la compréhension de l'écrit sont les domaines mineurs. Ce premier volume décrit le profil de compétence des élèves. Après avoir explicité les grands principes de l'enquête, le rapport analyse les performances des élèves en mathématiques selon les pays, en étudiant les variations par rapport à l'enquête de 2003, les répartitions aux différents niveaux de culture mathématique ou celles entre les sexes. Un chapitre examine comment l'exposition à des contenus mathématiques (possibilités d'apprentissage) est liée à la performance des élèves. Les deux derniers chapitres analysent les performances des élèves en compréhension de l'écrit et en sciences. 


\section{Innovative learning environments}

Paris : OCDE, 2013, 215 p.

L'innovation est un élément clé des sociétés et des économies d'aujourd'hui, et ceci inclut la façon dont nous apprenons. Ce rapport examine des environnements d'apprentissage innovants dans le cadre du projet Innovative learning environments (ILE) du Centre de recherche et d'innovation dans l'enseignement (CERI) de l'OCDE. Le projet a réuni 125 exemples de plus de vingt pays et des recherches ont été effectuées sur quarante d'entre eux pour fournir des études de cas détaillées. L'ouvrage, organisé en huit chapitres, se concentre sur quatre sources de changements essentiels: regroupement des enseignants et des autres éducateurs dans une même équipe; regroupement des apprenants au-delà des classes et des âges ; réexamen de l'utilisation du temps d'apprentissage et innovations en matière de pédagogie et d'évaluation.

UNESCO

Situacion educativa de América Latina y el Caribe: hacia la educación de calidad para todos al 2015

Santiago de Chile: UNESCO. Bureau régional pour l'éducation en Amérique latine et dans les Caraïbes, septembre 2013, 208 p. [en ligne]

Ce rapport analyse les progrès des pays d'Amérique latine et des Caraïbes (41 pays et territoires) en matière de réalisation des six objectifs de l'Éducation pour tous (EPT) fixés dans le Cadre d'action de Dakar en 2000. Au cours des années 2000, la plupart des pays de la région ont connu des progrès importants en matière économique et une évolution démographique permettant un développement de l'éducation. Toutefois, la persistance de niveaux élevés d'inégalité et de la pauvreté ainsi que la forte proportion de la population vivant dans les zones rurales freinent la réalisation d'une éducation de qualité pour tous. Le rapport passe en revue les résultats sur la décennie par niveaux (pré-primaire, primaire, secondaire, supérieur) et par grandes thématiques.

UNESCO

Rose Pauline (sous la direction de)

Rapport mondial de suivi sur l'EPT 2013/4 : enseigner et apprendre : atteindre la qualité pour tous

Paris : UNESCO, février 2014, 443 p. [en ligne]

Ce rapport annuel fait le bilan des progrès accomplis par les pays vers la réalisation des objectifs mondiaux de l'éducation adoptés en l'an 2000. Malgré les avancées de ces dix dernières années, aucun des objectifs de l'Éducation pour tous (EPT) ne sera atteint à l'échelle mondiale d'ici à 2015. 57 millions d'enfants ne sont toujours pas scolarisés et, qu'ils aient été scolarisés ou non, un tiers des enfants en âge de fréquenter l'enseignement primaire ne possède pas les compétences de base. Le rapport montre la nécessité de valoriser le potentiel des enseignants afin d'améliorer la qualité de l'apprentissage et considère nécessaire le recrutement d'1,6 million d'enseignants supplémentaires. 


\section{AUTEUR}

\section{BERNADETTE PLUMELLE}

Bernadette Plumelle est ingénieure de recherche et responsable du centre de ressources et d'ingénierie documentaires (CRID) du Centre international d'études pédagogiques (CIEP). 